\title{
NON-ZEEMAN CIRCULAR POLARIZATION OF MOLECULAR MASER SPECTRAL LINES
}

\author{
Martin Houde \\ Department of Physics and Astronomy, The University of Western Ontario, London, ON N6A 3K7, Canada \\ AND \\ Division of Physics, Mathematics and Astronomy, California Institute of Technology, Pasadena, CA 91125, USA \\ Received 2014 January 6; accepted 2014 September 4; published 2014 October 9
}

\begin{abstract}
We apply the anisotropic resonant scattering model developed to explain the presence of non-Zeeman circular polarization signals recently detected in the ${ }^{12} \mathrm{CO}(J=2 \rightarrow 1)$ and $(J=1 \rightarrow 0)$ transitions in molecular clouds to Stokes $V$ spectra of $\mathrm{SiO} v=1$ and $v=2,(J=1 \rightarrow 0)$ masers commonly observed in evolved stars. It is found that the observed antisymmetric "S"- and symmetric " $\cup$ "- or " $\cap$ "-shaped spectral profiles naturally arise when the maser radiation scatters off populations of foreground molecules located outside the velocity range covered by the background maser radiation. Using typical values for the relevant physical parameters, it is estimated that magnetic field strengths on the order of a few times $15 \mathrm{mG}$ are sufficient to explain the observational results found in the literature.
\end{abstract}

Key words: circumstellar matter - masers - polarization - stars: AGB and post-AGB

Online-only material: color figures

\section{INTRODUCTION}

The recent discovery of circular polarization in ${ }^{12} \mathrm{CO}$ spectral lines in molecular clouds (Houde et al. 2013; Hezareh et al. 2013) has opened up the possibility of developing a new method for mapping magnetic fields. Until now, the measurement of circular polarization in spectral line profiles in the denser parts of molecular clouds and star-forming regions have been limited to the few Zeeman-sensitive molecules that have line intensities strong enough to yield a measurable broadening in line profiles (e.g., OH and CN; see Güsten et al. 1994; Crutcher et al. 1999; Falgarone et al. 2008). It follows that further polarization measurements on strong maser lines from several molecular species have also been particularly useful for studies of this kind (e.g., $\mathrm{H}_{2} \mathrm{O}, \mathrm{CH}_{3} \mathrm{OH}$ class I and II, OH, and SiO; see Fish 2007; Watson 2009; Vlemmings 2012; Sarma 2012 for recent reviews). Zeeman measurements result in direct estimates of magnetic field strengths (usually the line of sight component). However, the very low levels of polarization thus detected in the corresponding Stokes $V$ spectra imply that Zeeman studies can, so far, only be realized on a relatively small number of sources. It also follows that despite the fact that all other techniques used to study magnetic fields in weakly ionized media, either based on polarimetry (e.g., Chandrasekhar \& Fermi 1953; Hildebrand et al. 2000, 2009; Heyer et al. 2008; Houde et al. 2011, 2009; Houde 2004) or not (Houde et al. 2000a, 2000b, 2001; Li \& Houde 2008), only give indirect information about magnetic fields, they have nonetheless played an essential role for such studies.

The ${ }^{12} \mathrm{CO}(J=2 \rightarrow 1)$ Stokes $V$ spectrum measured by Houde et al. (2013) at the peak intensity position in Orion KL, with the Caltech Submillimeter Observatory (Hezareh \& Houde 2010), showed a clear detection of polarization at levels on the order of $1 \%$ away from the line core. Although their proposed anisotropic resonant scattering process could account for these levels of polarization, there remained two issues that could not be satisfactorily assessed at the time. First, within the resonant scattering model the presence of circular polarization in spectral lines arises from the conversion of linearly polarized radiation emanating from a population of molecules in the background. This polarization transformation occurs when this incident radiation scatters off a second population of the same molecular species in the foreground; a process that could not be experimentally verified. Second, it was also found that the Stokes $V$ line shapes predicted by the resonant scattering model tend to display an intrinsic antisymmetric profile, as do the Zeeman effect and other scattering processes (e.g., Deguchi \& Watson 1985), while the observations of ${ }^{12} \mathrm{CO}(J=2 \rightarrow 1)$ in Orion KL did not.

The recent work of Hezareh et al. (2013) on SNR IC 443 (G) have, however, provided strong evidence that the circular polarization signals they detected in ${ }^{12} \mathrm{CO}(J=2 \rightarrow 1)$ and $(J=1 \rightarrow 0)$ over the whole source (spanning between $1^{\prime}$ to $2^{\prime}$ in size) indeed result from a conversion of linear to circular polarization. These observations were obtained with the IRAM $30 \mathrm{~m}$ telescope, where circular polarization was detected at levels of up to approximately $1 \%$, while linear polarization was generally weaker at a few tenths of a percent. In their analysis, Hezareh et al. (2013) compared the polarization angles (PAs) of the ${ }^{12} \mathrm{CO}$ linear polarization with those from a map of dust emission at $345 \mathrm{GHz}$ obtained with PolKa at APEX. The observed difference in PA between the two sets of data was perfectly consistent with what would be expected if the background ${ }^{12} \mathrm{CO}$ linear polarization (aligned with the dust polarization) was partially converted to circular polarization through the anisotropic resonant scattering process of Houde et al. (2013). That is, as this polarization conversion process takes place, the scattered and remaining ${ }^{12} \mathrm{CO}$ linear polarization is rotated away from the PA of the incident polarization (and therefore that of the dust) in a predictable manner. Accordingly, it was then found that although the alignment between co-located linear polarization vectors in the molecular and dust polarization maps was almost inexistent, the reinsertion of the measured circular polarization in the linear polarization signals (i.e., by reversing the conversion of linear to circular polarization) revealed a very strong agreement between the corrected ${ }^{12} \mathrm{CO}$ linear polarization vectors and those from the dust continuum. Still, it remained that the Stokes $V$ spectra measured 
in SNR IC 443 (G) did not reveal clear antisymmetric line profiles.

As explained in Houde et al. (2013), the shape of a given Stokes $V$ spectrum produced by the anisotropic resonant scattering process can be very difficult to predict for turbulent molecular lines, such as those measured in Orion KL and SNR IC $443(\mathrm{G})$. This is because there is not one well-defined propagation path for the detected radiation. That is, the nature of resonant scattering itself makes it likely that this radiation can emanate from several directions "behind" the scattering molecules before scattering into the telescope beam. It is also to be expected that background photons emanating from different paths will experience differing conditions that will affect their scattered amplitude and conversion to circular polarization (e.g., changes in the angle between the incident linear polarization and the magnetic field aligning the scattering molecules). It follows from these considerations that masers, in view of their nature and simpler propagation characteristics, may provide a particularly interesting test for Stokes $V$ line shapes predicted by the anisotropic resonant scattering model.

This is what we endeavor to accomplish in this paper, where we apply the model of Houde et al. (2013) to the problem of maser radiation and compare its predictions to observational results existing in the literature. More precisely, we aim to investigate whether the line shapes and intensities of Stokes $V$ spectra from $\mathrm{SiO} v=1$ and $v=2,(J=1 \rightarrow 0)$ masers (at $43.1 \mathrm{GHz}$ and $42.8 \mathrm{GHz}$, respectively) detected in the AGB star IK Tau by Cotton et al. (2011) can be readily reproduced by the model of Houde et al. (2013). In what follows, we present a brief review of the anisotropic resonant scattering process in Section 2, show results from numerical calculations tailored to the $\mathrm{SiO} 42.8 \mathrm{GHz}, v=2,(J=1 \rightarrow 0)$ maser line in Section 3, and discuss their comparison with the observations of Cotton et al. (2011) in Section 4. Finally, we end with a short summary in Section 5.

\section{LINEAR TO CIRCULAR POLARIZATION CONVERSION THROUGH ANISOTROPIC RESONANT SCATTERING}

Following Houde et al. (2013), we introduce an incident background radiation state $\left|\psi_{0}\right\rangle$ linearly polarized at an angle $\theta$ with the plane-of-the-sky component of the foreground magnetic field

$$
\left|\psi_{0}\right\rangle=\alpha_{0}\left|n_{\|}\right\rangle+\beta_{0}\left|n_{\perp}\right\rangle
$$

where $\alpha_{0}=\cos (\theta)$ and $\beta_{0}=\sin (\theta)$. The $n$-photon states $\left|n_{\|}\right\rangle$and $\left|n_{\perp}\right\rangle$ are orthonormal and polarized in directions parallel and perpendicular to the foreground magnetic field, respectively. We assume that the incident radiation characterized by Equation (1) emanates from a population of a given molecular species in the background medium while another population of the same molecule responsible for the scattering of this incident radiation coexists with, and is spatially aligned by, the foreground magnetic field. As was shown in Houde et al. (2013), after undergoing a large number of anisotropic resonant scattering events with the foreground population, the incident radiation is transformed to a new state

$$
|\psi\rangle \simeq \alpha_{0} e^{i \phi}\left|n_{\|}\right\rangle+\beta_{0}\left|n_{\perp}\right\rangle
$$

where $\phi$ is the relative phase shift induced between the two linear polarization states in the process. This final state of the scattered radiation is what we would seek to measure with a telescope. Accordingly, it is straightforward to show that the corresponding normalized Stokes parameters defining the complete polarization state of the scattered signal are ${ }^{1}$

$$
\begin{gathered}
q=\alpha_{0}^{2}-\beta_{0}^{2} \\
u=2 \alpha_{0} \beta_{0} \cos (\phi) \\
v=2 \alpha_{0} \beta_{0} \sin (\phi) .
\end{gathered}
$$

For these equations we chose a system of reference aligned with axes parallel and perpendicular to the foreground magnetic field. More precisely, the linear PA

$$
\chi=\frac{1}{2} \arctan \left(\frac{u}{q}\right)
$$

is measured from the axis defined by the orientation of the magnetic field. The Stokes parameters $q_{0}, u_{0}$, and $v_{0}(=0)$ of the incident background radiation (i.e., pre-scattering), as well as the incident linear PA $\chi_{0}$, are obtained by setting $\phi=0$ in Equations (3)-(5). We then find, in this particular reference frame, that

$$
\begin{gathered}
q=q_{0} \\
u=u_{0} \cos (\phi) \\
v=u_{0} \sin (\phi) .
\end{gathered}
$$

It thus becomes clear from these relations that as a relative phase shift $\phi$ builds up, linear polarization is transferred from the incident Stokes $u_{0}$ parameter to the scattered circular polarization Stokes $v$. The total amount of polarization is conserved in the process. It is important to note that the linear PA is also transformed with

$$
\tan (2 \chi)=\cos (\phi) \tan \left(2 \chi_{0}\right),
$$

i.e., as linear polarization is converted to circular polarization the linear PA rotates away from its original orientation.

This is the effect that was observed by Hezareh et al. (2013) for the ${ }^{12} \mathrm{CO}(J=2 \rightarrow 1)$ and $(J=1 \rightarrow 0)$ transitions in SNR IC $443(\mathrm{G})$, as previously mentioned. They were able to recover $\chi_{0}$ at every position on their maps by successively testing out different orientations for the foreground magnetic field. This allowed them to (1) single out new reference frames to calculate the Stokes parameters $q\left(=q_{0}\right)$ and $u$ through a rotation of their measured parameters (in the equatorial coordinate system, Stokes $v$ is independent of the reference frame), (2) calculate the relative phase shift with $\phi=\arctan (v / u)$ (see Equations (4) and (5)), (3) determine the incident Stokes parameter

$$
u_{0}=u \cos (\phi)+v \sin (\phi),
$$

\footnotetext{
1 We use the IAU convention for circular polarization, i.e., Stokes $v=\mathrm{RHC}-\mathrm{LHC}$, with RHC the right-handed circular polarization intensity having its electric vector rotating counter-clockwise as seen by the observer, etc., Houde et al. (2013) used the IEEE convention, which defines Stokes $v$ as the negative of the above.
} 
and finally (4) calculate $\chi_{0}$ with a relation similar to Equation (6) using $q_{0}$ and $u_{0}$. The correct orientation for the foreground magnetic field was readily identified when the new angles $\chi_{0}$ aligned to a very high degree with the PA obtained with their complementary dust polarization measurements. Hezareh et al. (2013) were thus able to confirm that the presence of circular polarization in their ${ }^{12} \mathrm{CO}$ spectral lines indeed originates from a conversion of incident background linear polarization.

The strength of the polarization conversion effect depends on the physical conditions found in both the background region where the incident linear polarized radiation emanates from and the volume occupied by the foreground scattering gas. It is therefore important to distinguish between the two corresponding sets of parameters entering in the different equations that follow. We also emphasize that the conditions found in the two regions (i.e., background and foreground) can be significantly different; this is certainly the case for the excitation temperatures and gas densities. This is especially important to keep in mind for the case treated in this paper since the incident radiation we will consider results from masers located in the background, implying, for example, the presence of a population inversion. The foreground molecular population is in no such way constrained. In fact, although unlikely to be perfectly realized, we will assume that local thermodynamic equilibrium (LTE) applies for the determination of level populations in the foreground scattering gas.

According to the anisotropic resonant scattering model of Houde et al. (2013), the relative phase shift can be evaluated as a function of the frequency $\omega$ of the incident radiation with

$$
\begin{aligned}
\phi(\omega) \simeq & \omega_{Z}^{2} \sin ^{2}(\iota) \tau \mathcal{V}_{\mathrm{int}} \frac{n g_{1} e^{-E_{\mathrm{l}} / k T_{\mathrm{ex}}}}{Q\left(T_{\mathrm{ex}}\right)} \\
& \times \frac{3 \pi c^{3} A_{\mathrm{ul}}}{4 \hbar \omega_{0}^{3} \omega^{2}} \sqrt{u(\omega) u^{\prime}(\omega)} I(\omega),
\end{aligned}
$$

where

$$
\begin{gathered}
I(\omega)=\int\left\{x^{2}(x-\omega)\left[3(x-\omega)^{2}-\gamma_{\mathrm{ul}}^{2}-\omega_{Z}^{2}\right] /\left[(x-\omega)^{2}+\gamma_{\mathrm{ul}}^{2}\right]\right. \\
\left.+(x-\omega)\left(\omega^{2}-3 x^{2}\right)+\gamma_{\mathrm{ul}}^{2}(3 x-\omega)+\omega_{Z}^{2}(x+\omega)\right\} \frac{h(x)}{\Delta} d x,
\end{gathered}
$$

with $\Delta=\left[\left(x+\omega_{Z}-\omega\right)^{2}+\gamma_{\mathrm{ul}}^{2}\right]\left[\left(x-\omega_{Z}-\omega\right)^{2}+\gamma_{\mathrm{ul}}^{2}\right]$. In Equations (12) and (13), $\omega_{0}$ is the frequency of the transition between the upper (u) and lower (l) energy levels in the rest frame of the scattering molecules (i.e., the frequency of the $\pi$-transition), $\omega_{Z}$ the Zeeman splitting due to the foreground magnetic field, $\gamma_{\mathrm{ul}}$ the total relaxation rate pertaining to the upper and lower states for the scattering molecules, $n$ their total volume density, $A_{\mathrm{ul}}$ the Einstein coefficient for the transition, $u(\omega)$ and $u^{\prime}(\omega)$ the energy densities of the incident and scattered radiation $\left(u(\omega)\right.$ corresponds to Stokes $u_{0}$ in the previous discussion), $h(x)$ the normalized spectral profile of the population of scattering molecules, and $\iota$ the inclination angle of the foreground magnetic field to the line of sight. The population of the lower state for the scattering molecules is calculated using the degeneracy $g_{1}$ and energy level $E_{1}$, while LTE at an excitation temperature $T_{\mathrm{ex}}$, at which the partition function $Q$ is evaluated, was assumed for the corresponding term in Equation (12). The volume of the region of interaction $\mathcal{V}_{\text {int }}$, where resonant scattering occurs, is constrained in directions perpendicular to the radiation's direction of propagation by the lifetimes of the two maser states through $l_{\gamma} \approx c / \gamma_{\mathrm{ul}}^{\prime}$, with $\gamma_{\mathrm{ul}}^{\prime}$ the total relaxation rate corresponding to the masing molecules. Along the direction of propagation, $\mathcal{V}_{\text {int }}$ is limited to the smallest of the physical size $l_{s}$ of the region harboring the scattering molecules and the effective mean path $l_{\mathrm{p}}$ of a photon. The time of interaction $\tau$ between the radiation and the molecules is set by $1 / \gamma_{\mathrm{ul}}^{\prime}$.

For the numerical calculations presented in the next section, we use the following relations for the total relaxation rate corresponding to the upper and lower states of the maser transition and the effective mean path of a photon

$$
\begin{gathered}
\gamma_{\mathrm{ul}}^{\prime}=\gamma_{\mathrm{coll}}+\frac{\gamma_{\mathrm{rad}}}{2} \\
l_{\mathrm{p}}=\left[\alpha_{\omega}\left(\alpha_{\omega}+\sigma_{\omega}\right)\right]^{-1 / 2},
\end{gathered}
$$

where the collision rate of a maser molecule with the (dominant) hydrogen molecules of density $n_{\mathrm{H}_{2}}$ is $\gamma_{\text {coll }}=n_{\mathrm{H}_{2}}\langle\sigma v\rangle$, with the momentum-rate transfer coefficient $\langle\sigma v\rangle$ evaluated at the kinetic temperature of the gas harboring the maser $T_{\text {kin }}$ (Shull $\&$ Draine 1990). The total radiative decay rate $\gamma_{\text {rad }}$ includes all the related rates for the lower and upper states of the background maser population taken separately, as well as the emission (spontaneous and stimulated) and absorption rates for radiative transitions between them (Letokhov \& Johansson 2009). We have a similar equation for $\gamma_{\mathrm{ul}}$, the relaxation rate of the scattering molecules, but using the corresponding hydrogen molecules density, excitation temperature $T_{\mathrm{ex}}$, and radiative rates at that location. The coefficients of absorption (Rybicki \& Lightman 1979) and resonant scattering (Grynberg et al. 2010) integrated over the foreground molecular population of the lower state are, respectively,

$$
\begin{gathered}
\alpha_{\omega}=\frac{n g_{\mathrm{u}} e^{-E_{\mathrm{l}} / k T_{\mathrm{ex}}}}{Q\left(T_{\mathrm{ex}}\right)} \frac{\pi^{2} c^{2} A_{\mathrm{ul}}}{\omega^{2}} \frac{\hbar \omega}{k T_{\mathrm{ex}}} h(\omega) \\
\sigma_{\omega}=\frac{n g_{1} e^{-E_{\mathrm{l}} / k T_{\mathrm{ex}}}}{Q\left(T_{\mathrm{ex}}\right)} \frac{3 c^{2}}{\omega^{2}} 4 \pi^{3} \gamma_{\mathrm{ul}}^{\prime} h(\omega) .
\end{gathered}
$$

We note, finally, that the term $\omega_{Z}^{2} \sin ^{2}(\iota)$ in Equation (12) makes clear the explicit dependency of the polarization conversion effect on the square of the strength of the plane-of-the-sky component of the foreground magnetic field. This will therefore be another parameter entering in the numerical calculations to follow.

\section{CIRCULAR POLARIZATION SPECTRAL LINE PROFILES OF SiO $v=2,(J=1 \rightarrow 0)$ MASERS}

In a recent study of $\mathrm{SiO} v=1$ and $v=2,(J=1 \rightarrow 0)$ masers (at $43.1 \mathrm{GHz}$ and $42.8 \mathrm{GHz}$, respectively) in the AGB star IK Tau Cotton et al. (2011) presented, among other results, several Stokes $V$ spectra obtained at high spatial resolution with the Very Long Baseline Array. Their results revealed high levels of circular polarization, often amounting to a significant fraction of the strong linear polarization detected at corresponding positions. It is expected that the large levels of linear polarization detected for these lines are probably a consequence of anisotropic pumping, as the they would otherwise require an implausible level of maser saturation (Watson 2009). Although some of the Stokes $V$ spectral lines reported by Cotton et al. (2011) displayed typical Zeemanlike antisymmetric "S"-shaped profiles, several symmetric 
“ $\cup$ "- or " $\cap$ "-shaped profiles were also observed. Considering these circular polarization line profiles and levels, Cotton et al. (2011) concluded that their observations could not be explained through the Zeeman effect and related models (Elitzur 1996). They further considered the alternative scenario of Wiebe \& Watson (1998) for the conversion of linear to circular polarization based on population imbalance (Deguchi \& Watson 1985), but were also unable to successfully account for their observations with this model.

In this section, we endeavor to test whether the anisotropic resonant scattering model of Houde et al. (2013) can reproduce the observations of Cotton et al. (2011). To do so implies establishing a mechanism through which symmetric " $U$ "- and " $\cap$ "-shaped Stokes $V$ profiles can be produced, in addition to the antisymmetric "S"-shaped. This problem was not resolved in Houde et al. (2013).

To that end, it is first instructive to explain how the antisymmetric profiles arises in the resonant scattering model. For this, we consider the basic resonance integral that arises from the interaction between an incident photon of frequency $\omega$ with scattering molecules for a given transition (e.g., the $\pi$-transition; see Equation (34) or Equation (40) of Houde et al. 2013),

$$
J(\omega) \simeq \int \frac{x-\omega}{(x-\omega)^{2}+\gamma_{\mathrm{ul}}^{2}} h(x) d x .
$$

We note from this equation that for a symmetric profile $h(x)$ the scattering amplitude will have differing signs for frequencies on opposite sides of its center. Accordingly, the amplitude becomes zero when $\omega$ is located right at the center of $h(x)$, as there is then a net cancellation of scattering from molecules residing at lower and higher frequencies (or velocities). The scattering amplitude will be non-zero for any other photon frequencies, being positive or negative depending whether $\omega$ is on the low or high side of $h(x)$, respectively. This behavior is responsible for the appearance of an antisymmetric " $S$ "-shaped line profile when the spectral distribution of the incident radiation $(u(\omega)$ in Equation (12)) is also symmetric about the center of $h(x)$.

We should also be aware, however, that the outcome would be completely different if the profiles of the incident radiation $u(\omega)$ and the scattering molecules population $h(x)$ overlap minimally in velocity space. Indeed, a " $\cup$ "- or " $\cap$ "-shaped scattered profile would result if $h(x)$ was entirely located on only one side of the incident radiation profile with little to no overlap. For the case of a maser, this implies a resonant scattering that is external to the masing process. That is, while molecules in the foreground residing at velocities covered by the maser could simply partake in the masing process, other molecules outside that velocity range would act as resonant scatterers. The remaining question is whether the scattering would be strong enough to efficiently convert incident linear polarization to circular polarization.

We use the model of Houde et al. (2013) summarized in Equations (12)-(17) above to determine whether it can produce Stokes $V$ spectra consistent with the observations of Cotton et al. (2011). For this, we have used the model developed by Decin et al. (2010) for the circumstellar envelope (CSE) of IK Tau. The following parameters were used for the background gas where the maser radiation originates (in the inner CSE): $n_{\mathrm{H}_{2}}=10^{10} \mathrm{~cm}^{-3}, T_{\text {kin }}=1000 \mathrm{~K}$, while in the foreground where the resonant scattering takes place (5 AU further in the CSE): $n_{\mathrm{H}_{2}}=7.5 \times 10^{8} \mathrm{~cm}^{-3}, T_{\mathrm{ex}}=700 \mathrm{~K}$, and $B=$ $15 \mathrm{mG}$. In both regions the $\mathrm{SiO}$ abundance relative to $\mathrm{H}_{2}$ was set to $1.6 \times 10^{-5}$. We then applied these to the $\mathrm{SiO}$ $v=2,(J=1 \rightarrow 0) 42.8 \mathrm{GHz}$ maser transition for which $g_{1}=1, g_{\mathrm{u}}=3, E_{1} / k=3527 \mathrm{~K}, Q\left(T_{\mathrm{ex}}\right)=1163$, and $A_{\mathrm{ul}}=3 \times 10^{-6} \mathrm{~s}^{-1}$ (Müller et al. 2001). The Landé factor (relative to the nuclear magneton) is $g_{\mathrm{SiO}} \simeq-0.154$ to -0.155 for $v=0,1$, and 2 (Davis \& Muenter 1974). The incident linear polarization radiation $u(\omega)$ was given a Gaussian profile centered at $0 \mathrm{~km} \mathrm{~s}^{-1}$, with a FWHM of $0.75 \mathrm{~km} \mathrm{~s}^{-1}$ and peak intensity of $1 \mathrm{Jy}$, approximately matching the profiles presented in Cotton et al. (2011) (see their Figure 5). Likewise, we also used a Gaussian profile $h(x)$ for two populations of scattering molecules of $1.5 \mathrm{~km} \mathrm{~s}^{-1}$ width (FWHM), centered at velocities (or frequencies) located at $\pm 3 \mathrm{~km} \mathrm{~s}^{-1}$. These distributions for the scattering molecules approximately match the gas velocity profile at that position in the CSE, as determined in Decin et al. (2010). Depending on the cases studied in Figure 1, each population component can have a density $n=0,0.5$, or 1 times $1.2 \times 10^{3} \mathrm{~cm}^{-3}$. We use a value $5 \mathrm{~s}^{-1}$ for the radiative decay rates of the (vibrationally excited) lower and upper states and for the maser radiation we assume that the rate of stimulated emission is approximately equal to these decay rates (Wiebe \& Watson 1998; Watson 2009). Given these figures, and assuming a similar rate of absorption transitions from the lower to the upper state, we find that the collision rates are not significant in the calculations of $\gamma_{\mathrm{ul}}$ and $\gamma_{\mathrm{ul}}^{\prime}$, and even more so the spontaneous emission rate $A_{\mathrm{ul}}$. We thus calculate that $l_{\gamma} \approx c / \gamma_{\mathrm{ul}}^{\prime} \approx 2.3 \times 10^{9} \mathrm{~cm}$. The absence of a significant overlap between $u(\omega)$ and $h(x)$ (see Equations (15)-(17)) implies that the volume of the region of interaction cannot be constrained along the direction of propagation by the mean path $l_{p}$, as it then becomes exceedingly large. Lacking information on the physical size of the scattering region, we adopt the value $l_{s}=5 \mathrm{AU}$ $\left(7.5 \times 10^{13} \mathrm{~cm}\right)$. This does not appear to be an unreasonable or excessive size since it is known that the $\mathrm{SiO}$ maser ring (located inside the inner dust condensation zone) observed by Cotton et al. (2011) has a diameter of approximately 8 AU (i.e., 0.03 at a distance of $265 \mathrm{pc}$ ), while the $11 \mu \mathrm{m}$ interferometry observations of Hale et al. (1997) revealed a succession of more distant circumstellar shells surrounding IK Tau at radii of approximately 25 to $150 \mathrm{AU}$ (i.e., $\sim 0^{\prime} .1$ to $0^{\prime} .57$ at the same distance).

The results of our calculations are shown in Figure 1, where the circular polarization spectra of the $\mathrm{SiO}(v=2, J=1 \rightarrow 0)$ transition at $42.8 \mathrm{GHz}$ is shown for five different scattering populations. In all cases, the left panel shows the relative phase shift $\phi_{\mathrm{SiO}}$ calculated with Equation (12) (solid curve; using the vertical scale on the left), the incident linear polarization energy density $(u(v)$, dashed curve; using the vertical scale on the right), and the populations of scattering molecules $(h(v)$, dot-dashed and hashed curves; normalized for display purposes). The right panels show the resulting Stokes $V$ profile (solid curve) and, once more, the incident linear polarization energy density $(u(v)$, dashed curve); both use the vertical scale on the right. All quantities are plotted as a function of the velocity $v$. We also set the magnetic field in the plane of the sky (i.e., $\iota=\pi / 2$ ), $2 \alpha_{0} \beta_{0}=1$ (i.e., $\theta=\pi / 4$, resulting in $q_{0}=0$ and $u_{0}=1$ ), and $u(v) \simeq u^{\prime}(v)$ for simplicity in computing $\phi_{\mathrm{SiO}}$. The computed Stokes $V$ spectra are therefore of the form

$$
V(v) \propto e^{-\frac{1}{2}\left(\frac{v}{\Delta v}\right)^{2}} \sin \left[\phi_{\mathrm{SiO}}(v)\right],
$$

where the Gaussian profile is associated with $u(v)$.

The top panel of Figure 1 shows a clear example of a symmetric " $\cup$ "-shaped Stokes $V$ profile resulting from a minimal overlap in velocity space between the population of scattering molecules and the incident radiation. In this case, the foreground 


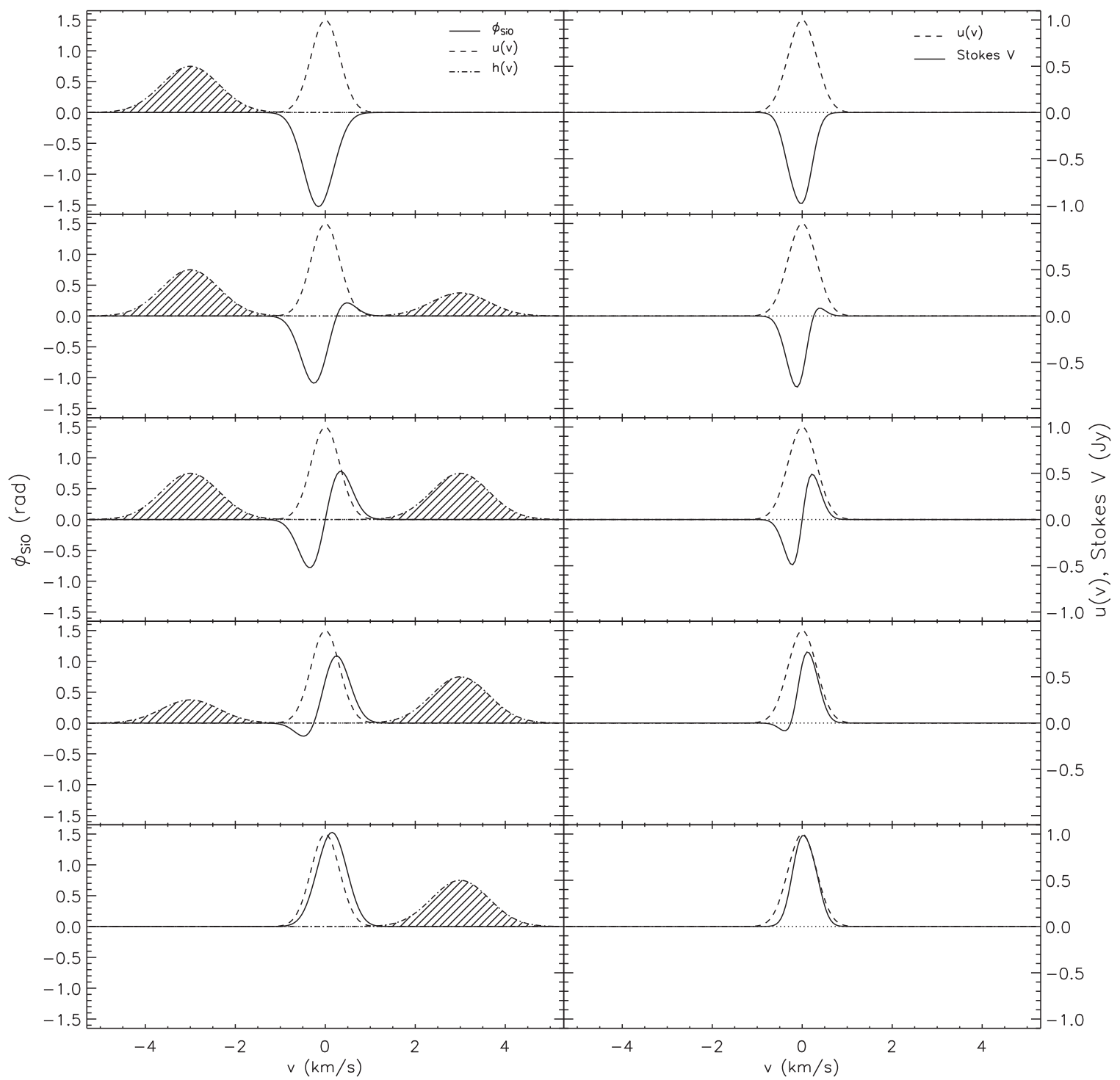

Figure 1. Circular polarization spectra of the $\mathrm{SiO}(v=2, J=1 \rightarrow 0)$ transition at $42.8 \mathrm{GHz}$ using the anisotropic resonant scattering model of Houde et al. (2013). The left panels show the relative phase shift $\phi_{\mathrm{SiO}}$ between the linear polarization components parallel and perpendicular to the orientation of the magnetic field in the plane of the sky (solid curve; using the vertical scale on the left), the incident linear polarization energy density $(u(v)$, dashed curve; using the vertical scale on the right), and the population of scattering molecules $(h(v)$, dot-dashed and hashed curve; normalized for display purposes). These were calculated using the model of Decin et al. (2010) for the CSE of IK Tau. For the background gas where the maser radiation originates (in the inner CSE), we set $n_{\mathrm{H}_{2}}=10^{10} \mathrm{~cm}^{-3}, T_{\mathrm{kin}}=1000 \mathrm{~K}$, while in the foreground where the resonant scattering takes place ( $5 \mathrm{AU}$ further in the CSE) we have $n_{\mathrm{H}_{2}}=7.5 \times 10^{8} \mathrm{~cm}^{-3}, T_{\mathrm{ex}}=700 \mathrm{~K}$, and $B=15 \mathrm{mG}$. In both regions the $\mathrm{SiO}$ abundance relative to $\mathrm{H}_{2}$ was set to $1.6 \times 10^{-5}$. The foreground $\mathrm{SiO}$ scattering populations therefore have a maximum density $n_{\mathrm{SiO}}=1.2 \times 10^{3} \mathrm{~cm}^{-3}$ for any of the two velocity ranges (i.e., centered at $\pm 3 \mathrm{~km} \mathrm{~s}^{-1}$ ). The right panels show the resulting Stokes $V$ profile (solid curve) and, once more, the incident linear polarization energy density $\left(u(v)\right.$, dashed curve); both curves use the vertical scale on the right. An interaction length $l_{s}=5$ AU were used for these calculations.

$\mathrm{SiO}$ molecules (hashed profile) are centered at $-3 \mathrm{~km} \mathrm{~s}^{-1}$ only and have a total density of $1.2 \times 10^{3} \mathrm{~cm}^{-3}$. As can be seen, even a relatively weak magnetic field of $15 \mathrm{mG}$ is sufficient to bring a significant relative phase shift of approximately $-1.5 \mathrm{rad}$ at $\simeq-0.2 \mathrm{~km} \mathrm{~s}^{-1}$. The resulting Stokes $V$ intensity profile (right panel) is almost as strong as the incident linear polarization signal. In the second panel from the top, an additional scattering population is added at $3 \mathrm{~km} \mathrm{~s}^{-1}$ but at half the density (i.e., $0.6 \times 10^{3} \mathrm{~cm}^{-3}$ ). This foreground population provides scattering of opposite sign and is therefore responsible for the appearance of a "positive" shoulder on the red side of the $\phi_{\mathrm{SiO}}$ and Stokes $V$ profiles. The middle panel has equal foreground molecular populations on either side of the incident radiation spectrum resulting in antisymmetric " $\mathrm{S}$ "-shaped $\phi_{\mathrm{SiO}}$ and Stokes $V$ profiles, reminiscent of a typical Zeeman circular polarization spectrum. The trend is reversed in the last two panels where the population at $3 \mathrm{~km} \mathrm{~s}^{-1}$ becomes increasingly more important in similar proportions, yielding analogous but inverted profiles. We therefore find that both antisymmetric "S"- and symmetric " $U$ "or " $\cap$ "-shaped Stokes $V$ profiles naturally arise from the model of Houde et al. (2013), when anisotropic resonant scattering is allowed to occur externally to the maser (in velocity space).

Similar results are obtained for masers at the $\mathrm{SiO}(v=1$, $J=1 \rightarrow 0$ ) transition at $43.1 \mathrm{GHz}$.

\section{DISCUSSION}

The results of our numerical calculations shown in Figure 1 establish that the anisotropic resonant scattering model of Houde et al. (2013) provides a viable explanation for the IK Tau $\mathrm{SiO}$ $v=1$ and $v=2,(J=1 \rightarrow 0)$ masers observations of 
Cotton et al. (2011). The key to reproducing the observed Stokes $V$ profiles is the realization that resonant scattering can occur from the interaction of the maser radiation with populations of foreground molecules located outside the velocity range covered by the maser. As is clearly demonstrated in Figure 1, depending on the arrangement of the relative populations on either side of the maser line, a sequence of symmetric " $U$ "- or " $\cap$ "- and antisymmetric " $S$ "-shaped spectral profiles naturally arises. These Stokes $V$ spectra share the same characteristics as those seen in Cotton et al. (2011) (see their Figure 5, for example). For IK Tau the overall population of $\mathrm{SiO}$ molecules emits at velocities covering from approximately $26-40 \mathrm{~km} \mathrm{~s}^{-1}$ (see Figure 2 in Cotton et al. 2011), with narrow maser features interspersed over that range. The possibility for maser radiation to interact with molecules located at neighboring velocities is therefore non-negligible. This is also consistent with the mapping of $22 \mathrm{GHz}$ water masers in this source (and others) by Richards et al. (2012), where overdense regions of gas containing a significant fraction of the mass loss are seen to be located over a large range of distances from the center of expansion. These clumps have sizes and velocities that scale with their positions in the CSE and, although they were mapped with water masers, are likely to also contain $\mathrm{SiO}$ gas. For IK Tau, in particular, these clouds have radii measured to be on the order of 1-2 AU at a distance of a few astronomical units from the expansion center. We also note that Leal-Ferreira et al. (2013) have successfully detected circular polarization signals in such overdense gas regions in IK Tau (and other sources) using the $22 \mathrm{GHz}$ water maser line. The observed Stokes $V$ spectra are consistent with a simple Zeeman model (i.e., they display an anti-symmetric "S" profile) and yield magnetic field strengths on the order of $100 \mathrm{mG}$, when thus interpreted.

Although there are significant uncertainties on the parameters used in our calculations (e.g., temperatures, densities, and the volume of the region of interaction $\mathcal{V}_{\text {int }}=l_{\gamma}^{2} l_{s}$ ), the model is relatively robust in reproducing the results as there exists a significant domain of combinations that can lead to similar conclusions. For example, the results shown in Figure 1 obtained with the following parameters in the foreground where the polarization conversion takes place: $n_{\mathrm{H}_{2}}=7.5 \times 10^{8} \mathrm{~cm}^{-3}$, $T_{\mathrm{ex}}=700 \mathrm{~K} B=15 \mathrm{mG}$, and $l_{s}=5 \mathrm{AU}$ apply equally well for $n_{\mathrm{H}_{2}}=7.5 \times 10^{8} \mathrm{~cm}^{-3}, B \simeq 50 \mathrm{mG}$, and $l_{s}=$ $0.5 \mathrm{AU}$ or $n_{\mathrm{H}_{2}}=7.5 \times 10^{7} \mathrm{~cm}^{-3}, B \simeq 50 \mathrm{mG}$, and $l_{s}=$ $5 \mathrm{AU}$, etc. Likewise, displacing the populations of foreground molecules from $\pm 3 \mathrm{~km} \mathrm{~s}^{-1}$ to $\pm 4 \mathrm{~km} \mathrm{~s}^{-1}$ only requires increasing the magnetic field strength from $15 \mathrm{mG}$ to $30 \mathrm{mG}$ to preserve the intensity and shape of the Stokes $V$ spectra. Furthermore, the exact shape of the scattering population distributions is largely irrelevant, and only their numbers (i.e., densities) matter. It is interesting to compare these magnetic field strengths with those needed for the simple Zeeman model (i.e., 1.9-6 G for IK Tau; Herpin et al. 2006, or more in some cases; Watson 2009) to match the measured levels of circular polarization (up to a few tens of percent; see Figure 12 in Cotton et al. 2011). The amount of circular polarization predicted by the scattering model is only limited by that of the incident linear polarization; it is clear from our results of Figure 1 and Equation (12) that a minimal increase in magnetic field strength or adjustment in other parameters could lead to a complete conversion of the linear polarization. Although it is to be noted that gradients in the velocity of the gas and magnetic field strength along the line of sight can account for deviations from the usual antisymmetric "S" shape of Stokes
$V$ Zeeman spectra (for Zeeman sensitive species, especially, this can even lead to the "disappearance" of spectral components; Watson 2009), the fact that the simple Zeeman model cannot reproduce the symmetric Stokes $V$ profiles observed by Cotton et al. (2011) renders our model an even more viable alternative for explaining the polarization characteristics of $\mathrm{SiO}$ masers in evolved stars. This is because, as explained by Cotton et al. (2011), the lack of significant velocity structure for neighboring lines of sight in relation to the overall range of velocities where masers were detected makes it unlikely that such effects can explain their observations. In a more general context, we should also note that based on the strength of magnetic fields predicted and observed alone, a hybrid of Zeeman and scattering nonZeeman process to explain the observed circular polarization spectra should not be ruled out. For example, the field strength of a few times $15 \mathrm{mG}$ at $10 \mathrm{AU}$ in the CSE of IK Tau needed for the resonant scattering model could imply the presence of a surface magnetic field of the order of $1 \mathrm{G}$ when we adopt a $1 / r^{2}$ functional form for the magnetic field strength as a function of position in the CSE. This would not necessarily be inconsistent with other results from maser measurements. It is also interesting to note that a line-of-sight surface magnetic field strength of 2-3 G has recently been estimated from circular polarization measurements for another Mira star (i.e., $\chi$-Cigni) by Lèbre et al. (2014). Although this value is consistent with our previous estimate using our calculations for the $\mathrm{SiO}$ lines in IK Tau, Lèbre et al. (2014) concluded, however, that their results were more consistent with a $1 / r$ than a $1 / r^{2}$ magnetic field strength scaling.

On the other hand, it must be acknowledged that the models presented here are probably too simple to fully account for the complexity of the physical conditions found in the CSE of IK Tau (or other objects in general), as well as for the range of existing observations. One notable example concerning the observations of Cotton et al. (2011) is the case of Regions B and $\mathrm{C}$ for their $\mathrm{SiO} v=2,(J=1 \rightarrow 0) 42.8 \mathrm{GHz}$ observations. Considering that the two masers occur at the same velocity (i.e., $\simeq 39 \mathrm{~km} \mathrm{~s}^{-1}$ ) and that they are spatially separated by approximately $1 \mathrm{AU}$ (as projected on the sky; see Figure 4 of Cotton et al. 2011), the apparent similarity in their environments may make it difficult to reconcile the fact that their Stokes $V$ spectra are inverted from one another (see Figure 5 of Cotton et al. 2011). That is, the Stokes $V$ spectrum of Region B is similar to the top spectrum in our Figure 1, while that of Region $\mathrm{C}$ resembles the bottom spectrum. We can put forth a simple solution to this problem by noting that an inversion of a given Stokes $V$ spectrum does not have to result from a displacement of the scattering foreground molecules from, say, the low side to the high side of the incident radiation in velocity space, but can also result from a change in the relative alignment between the linear polarization state of the incident radiation and the foreground magnetic field. Since the overall sign of the spectrum is controlled through the $2 \alpha_{0} \beta_{0}=\sin (2 \theta)$ factor in Equation (5), the needed angular rotation for the sign change can be small if $\theta$ is small to start with, or at most as large as $\pi / 4$ if $\theta$ is initially close to $\pi / 4$. Furthermore, these potential changes in $\theta$ may also be required in a more general sense since it is unlikely that $\mathrm{SiO}$ maser radiation blueshifted relative to the velocity center can interact with both relatively blue- and redshifted scattering gas in the CSE, as implied in our Figure 1. That is, since the scattering gas must be located between the maser formation region and the observer, the nature of the expanding wind in the CSE imply that a blueshifted maser 


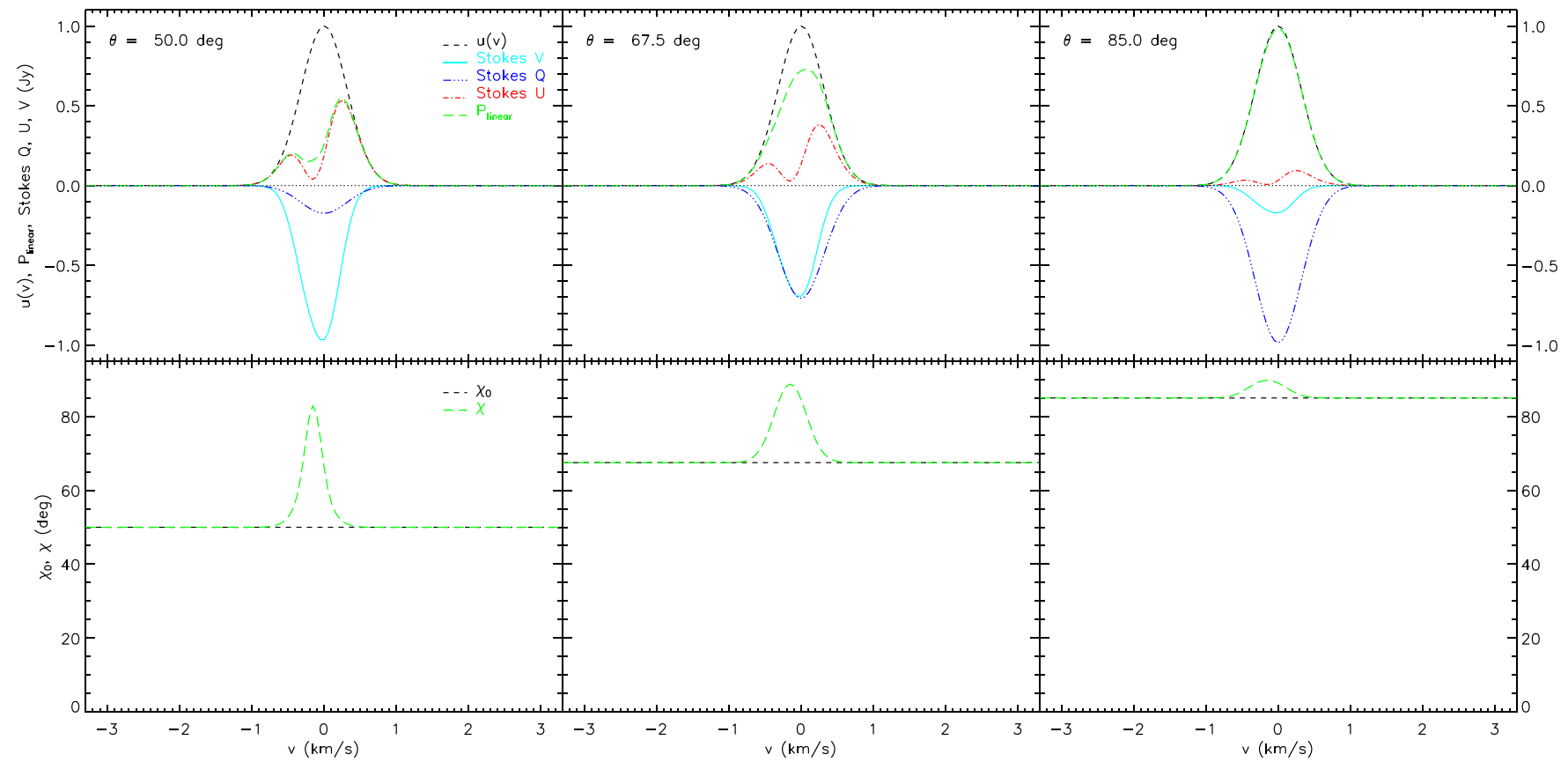

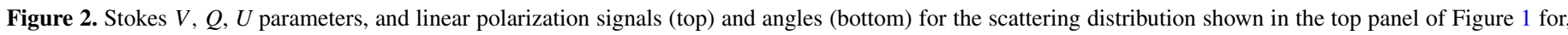

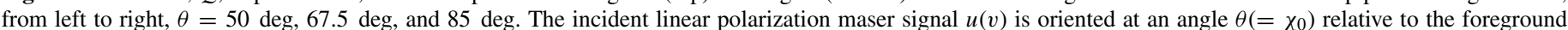

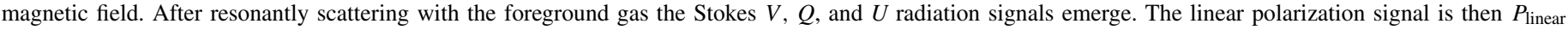
oriented at an angle $\chi$ relative to the foreground magnetic field (positive Stokes $Q$ is aligned with the magnetic field).

(A color version of this figure is available in the online journal.)

radiation reaching the observer can only resonantly scatter with gas that is further blueshifted in velocity space. Evidently, the presence of turbulent, infall, or rotational velocity components may alter this picture. Nonetheless, changes in the relative orientation between the foreground magnetic field and the linear polarization state of the incident maser radiation for different pockets of scattering gas along the line of sight may therefore be needed to account for all observations. For redshifted maser emission, it is also possible that blue- and redshifted scattering gas would not be subjected to the same physical conditions in view of their respective locations in the CSE. For simplicity, we have not accounted for these differences in our calculations. In all cases, the added complexity needed in our model resides in accurately knowing a set of physical parameters to a level of precision we do not possess.

Incidentally, the fact that redshifted masers can scatter with both relatively blue- and redshifted gas and have to propagate through a larger column of gas in the CSE before reaching the observer in comparison to blueshifted masers (i.e., they are located further away along the line of sight) would lead us to expect that the presence of circular polarization should be more prevalent in the spectra of redshifted masers. It is therefore interesting to note that most of the spectra for the two transitions presented in Cotton et al. (2011) are redshifted (i.e., five out of six for each transition, only Regions $\mathrm{F}$ are blueshifted).

We also note that a previous observational comparison of circular polarization levels between the $43.1 \mathrm{GHz}(v=1, J=$ $1 \rightarrow 0)$ and $86.2 \mathrm{GHz}(v=1, J=2 \rightarrow 1) \mathrm{SiO}$ maser lines for VY CMa (McIntosh et al. 1994) revealed that a corresponding ratio of the Stokes $V$ intensities between the two spectra of $\sim 2$, consistent with the Zeeman effect. A similar argument can be made through a comparison of the results of Herpin et al. (2006) for IK Tau for the $86.2 \mathrm{GHz}(v=1, J=2 \rightarrow 1)$ line with those of Cotton et al. (2011) at $43.1 \mathrm{GHz}(v=1, J=1 \rightarrow 0)$. That is, Herpin et al. (2006) find the average ratio of circular to linear polarization to be $\sim 0.3$ at the higher frequency, while Cotton et al. (2011) measure it to be 0.13 at $43.1 \mathrm{GHz}$. We again find a ratio neighboring 2 favoring the lower frequency transition. We performed similar calculations as those shown in Figure 1 for these two $v=1$ transitions, as well as for the $85.6 \mathrm{GHz}$ $(v=2, \quad J=2 \rightarrow 1)$ line since our previous calculations of Figure 1 were made for the $42.8 \mathrm{GHz}(v=2, J=1 \rightarrow 0)$ line, and compared the Stokes $V$ intensities. The ratio of the $(J=1 \rightarrow 0)$ to $(J=2 \rightarrow 1)$ intensities varies somewhat depending on the case chosen (i.e., which panel of Figure 1 is used), but we find that it is also close to 2 for both pairs of lines. For example, the $42.8 \mathrm{GHz} / 85.6 \mathrm{GHz}$ ratio for the top panel of Figure 1 the peak Stokes $V$ intensity numbers are $\simeq-1:-0.6$, while for the middle panel they are $\simeq 0.49: 0.23$. It thus appears that the ratios obtained for the resonant scattering model are also consistent with the aforementioned observations.

A further test that can be applied to our model consists of producing corresponding linear polarization spectra to the results shown in Figure 1. This was done using Equations (3)-(6) and the resulting spectra are shown in Figures 2-4. Care must be taken when comparing these spectra with the observations of Cotton et al. (2011) since their spectra are those obtained after resonant scattering has taken place. The amount of rotation induced on the linear polarization signal is a function of the amount of scattering taking place (in the phase shift $\phi$ of Equation (10)), as well as the initial orientation of the incident linear polarization in relation to the foreground magnetic field before scattering (i.e., the angle $\theta$ ). Because we do not a priori know these two orientations, it is impossible to determine the precise conditions that led to the spectra presented in Cotton et al. (2011). Still, it may be possible to test, under simple assumptions, whether some spectral features predicted by our model are realized observationally. 


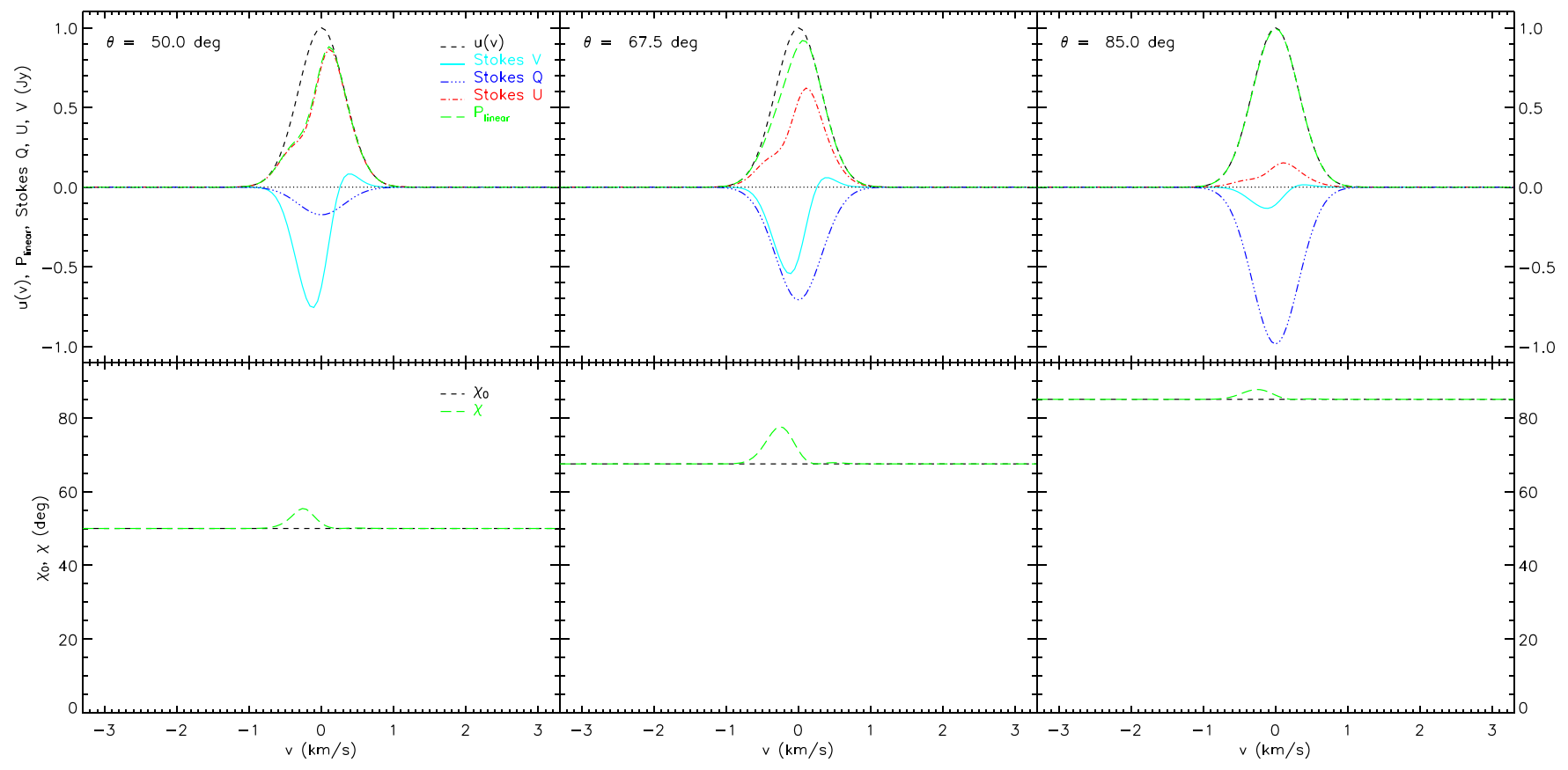

Figure 3. Same as Figure 2, but for the case corresponding to the second panel from the top of Figure 1.

(A color version of this figure is available in the online journal.)

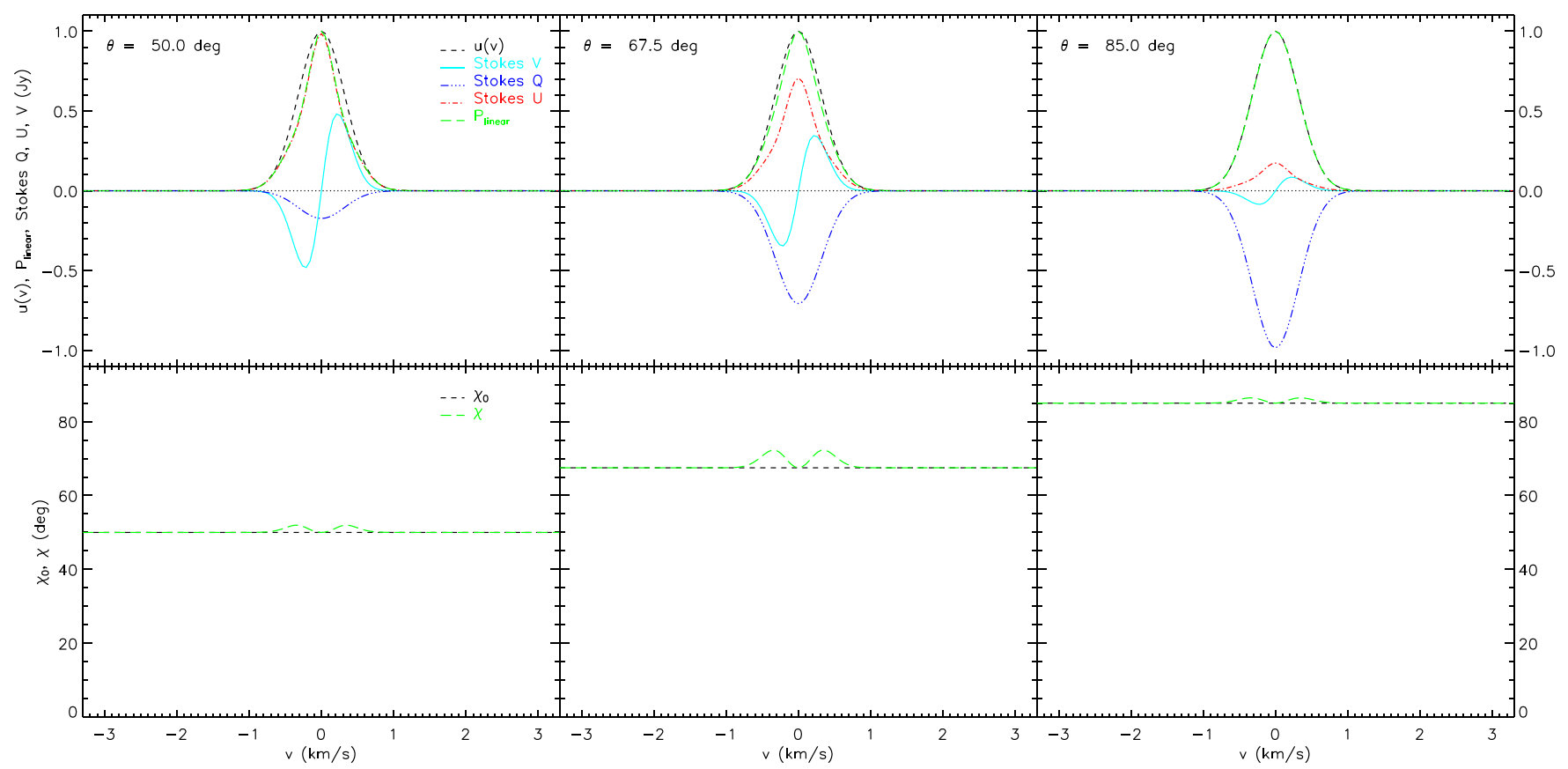

Figure 4. Same as Figure 2, but for the case corresponding to the middle panel of Figure 1.

(A color version of this figure is available in the online journal.)

More precisely, for the results presented in Figures 2-4 we have produced the Stokes $V, Q$, and $U$ spectra, as well as calculated the linear polarization $P_{\text {linear }}=\left(Q^{2}+U^{2}\right)^{1 / 2}$ and angles $\chi=0.5 \arctan (U / Q)$ for different relative orientations between the incident linear polarization (of amplitude $u(v)$ and angle $\chi_{0}$ in the figures) and the foreground magnetic field. These orientations were set at $\theta=50,67.5$, and $85 \mathrm{deg}$. At $\theta=45 \mathrm{deg}$, all of the incident linear polarization is in Stokes $U_{0}$ and the conversion effect will most strongly affect the shape of $P_{\text {linear }}$, however, the angle $\chi$ will not show any features since Stokes $Q$ is then zero; choosing a slightly displaced orientation at $\theta=50 \mathrm{deg}$ allows us to better observe the effect in that neighborhood. Conversely, the conversion effect is inexistent at $\theta=90 \mathrm{deg}$ since then all of the incident linear polarization is in Stokes $Q_{0}$; we therefore chose $\theta=85 \mathrm{deg}$. Most importantly, we emphasize that the angle $\theta$ was assumed constant across the spectral line. Evidently, this not need to be the case in reality, and variations in $\theta$ as a function of velocity would alter the spectral shapes produced in our calculations.

Figure 2 corresponds to the top panel of Figure 1 (symmetric Stokes $V$ ), while Figures 3 and 4 are for the second (from the top) and middle panels (anti-symmetric Stokes $V$ ). One can see that 
significant deformations in the linear polarization spectra $P_{\text {linear }}$ can be observed when most of the incident linear polarization is in Stokes $U$ for the cases of symmetric Stokes $V$ profiles (see $\theta=50 \mathrm{deg}$ in Figure 2). In that case, a "blue" shoulder appears in the linear polarization spectrum. We note that some of Cotton et al. (2011) spectra appear to share some of that characteristic (e.g., Region D in their Figure 5). On the other hand, the PA $\chi$ for that same case shows important variations over a larger range of orientations $\theta$ (e.g., several tens of degrees when $\theta=50$ and $\theta=67.5 \mathrm{deg}$ in Figure 2). Again, some of the spectra of Cotton et al. (2011) also display large variations in PAs (sometimes more severe), although it is difficult to pick a specific pattern from their data. The same types of behavior are observed in Figure 3 but to a lesser degree.

The anti-symmetric Stokes $V$ case of Figure 4 shows that the shape of the linear polarization spectrum $P_{\text {linear }}$ is not significantly impacted by the presence of circular polarization for the set of parameters used in our calculations. Interestingly, the corresponding pattern for the PA $\chi$ is similar in shape to the one found for Region A in Figure 5 of Cotton et al. (2011). This is more easily seen through a comparison with the middle panel of Figure 4 when $\theta=67.5 \mathrm{deg}$. The amplitude of the variation could to some extent be adjusted by tuning the parameters (e.g., slightly changing the strength of the magnetic field). Also, the range of values for $\chi$ could be shifted up or down by a simple rotation of the reference frame used to calculate Stokes $Q$ and $U$ with respect to the orientation of the foreground magnetic field.

It remains to generalize and apply the anisotropic resonant scattering model to other maser transitions such as for water and methanol, which, unlike $\mathrm{SiO}$, are asymmetric top molecules. It will be interesting to find out if, for example, the polarization characteristics of the $6.7 \mathrm{GHz}$ methanol maser can be satisfactorily reproduced. Given the current uncertainty concerning the Zeeman sensitivity of that transition (Vlemmings et al. 2011), physical models requiring weaker magnetic field strengths may become necessary.

\section{CONCLUSION}

In this paper, we applied the anisotropic resonant scattering model developed by Houde et al. (2013) to explain the presence of non-Zeeman circular polarization signals recently detected in ${ }^{12} \mathrm{CO}(J=2 \rightarrow 1)$ and $(J=1 \rightarrow 0)$ transitions in molecular clouds (Houde et al. 2013; Hezareh et al. 2013) to Stokes $V$ spectra of $\mathrm{SiO} v=1$ and $v=2,(J=1 \rightarrow 0)$ masers commonly observed in evolved stars. It was found that the observed antisymmetric " $S$ "- and symmetric " $\cup$ "- or " $\cap$ "shaped spectral profiles naturally arise when the maser radiation scatters off populations of foreground molecules located outside the velocity range covered by the maser. Using typical values for the relevant physical parameters, it is estimated that magnetic field strengths on the order of a few times $15 \mathrm{mG}$ are sufficient to explain the observational results previously published by Cotton et al. (2011) for the evolved star IK Tau.

We are grateful to T. Hezareh and H. Wiesemeyer for helpful discussions. M.H.'s research is funded through the NSERC
Discovery Grant, Canada Research Chair, and Western's Academic Development Fund programs.

\section{REFERENCES}

Chandrasekhar, S., \& Fermi, E. 1953, ApJ, 118, 113

Cotton, W. D., Ragland, S., \& Danchi, W. C. 2011, ApJ, 736, 96

Crutcher, R. M., Troland, T. H., Lazareff, B., Paubert, G., \& Kazès, I. 1999, ApJL, 514, L121

Davis, R. E., \& Muenter, J. S. 1974, JChPh, 61, 2940

Decin, L., De Beck, E., Brünken, S., et al. 2010, A\&A, 516, 69

Deguchi, S., \& Watson, W. D. 1985, ApJ, 289, 621

Elitzur, M. 1996, ApJ, 457, 415

Falgarone, E., Troland, T. H., Crutcher, R. M., \& Paubert, G. 2008, A\&A, 487,247

Fish, V. L. 2007, in IAU Symp. 242, Astrophysical Masers and Their Environments, ed. J. M. Chapman \& W. A. Baan (Cambridge: Cambridge Univ. Press), 71

Grynberg, G., Aspect, A., \& Fabre, C. 2010, Introduction to Quantum Optics (Cambridge: Cambridge Univ. Press)

Güsten, R., Fiebig, D., \& Uchida, K. I. 1994, A\&A, 286, L51

Hale, D. D. S., Bester, M., Danchi, W. C., et al. 1997, ApJ, 490, 407

Herpin, F., Baudry, A., Thum, C., Morris, D., \& Wiesemeyer, H. 2006, A\&A 450, 667

Heyer, M., Gong, H., Ostriker, E., \& Brunt, C. 2008, ApJ, 680, 420

Hezareh, T., \& Houde, H. 2010, PASP, 122, 786

Hezareh, T., Wiesemeyer, H., Houde, M., Gusdorf, A., \& Siringo, G. 2013, A\&A, 558, 45

Hildebrand, R. H., Davidson, J. A., Dotson, J. L., et al. 2000, PASP, 112,1215

Hildebrand, R. H., Kirby, L., Dotson, J. L., Houde, M., \& Vaillancourt, J. E. 2009, ApJ, 696, 567

Houde, M. 2004, ApJL, 616, L111

Houde, M., Bastien, P., Peng, R., Phillips, T. G., \& Yoshida, H. 2000a, ApJ, 536,847

Houde, M., Hezareh, T., Jones, S. C., \& Rajabi, F. 2013, ApJ, 764, 24

Houde, M., Peng, R., Phillips, T. G., Bastien, P., \& Yoshida, H. 2000b, ApJ, 537,245

Houde, M., Phillips, T. G., Bastien, P., Peng, R., \& Yoshida, H. 2001, ApJ, 547,311

Houde, M., Rao, R., Vaillancourt, J. E., \& Hildebrand, R. H. 2011, ApJ, 733, 109

Houde, M., Vaillancourt, J. E., Hildebrand, R. H., Chitsazzadeh, S., \& Kirby, L. 2009, ApJ, 706, 1504

Leal-Ferreira, M. L., Vlemmings, W. H. T., Kemball, A., \& Amiri, N. 2013, A\&A, 554, 134

Lèbre, A., Aurière, M., Fabas, N., et al. 2014, A\&A, 561, 85

Letokhov, V., \& Johansson, S. 2009, Astrophysical Lasers (New York: Oxford Univ. Press)

Li, H., \& Houde, M. 2008, ApJ, 677, 1151

McIntosh, G. C., Predmore, C. R., \& Patel, N. A. 1994, ApJL, 428, L29

Müller, H. S. P., Thorwith, S., Roth, D. A., \& Winnewisser, G. 2001, A\&A, 370, L49

Richards, A. M. S., Etoka, S., Gray, M. D., et al. 2012, A\&A, 546, 16

Rybicki, G. B., \& Lightman, A. P. 1979, Radiative Processes in Astrophysics (New York: Wiley)

Sarma, A. P. 2012, in IAU Symp. 287, Cosmic Masers: from $\mathrm{OH}$ to $\mathrm{H}_{0}$, ed. R. S. Booth, E. M. L. Humphreys, \& W. H. T. Vlemmings (Cambridge: Cambridge Univ. Press), 41

Shull, J. M., \& Draine, B. T. 1990, in Interstellar Processes, ed. D. J. Hollenback \& H. A. Thronson, Jr. (Dordrecht: Kluwer), 225

Vlemmings, W. H. T. 2012, in IAU Symp. 287, Cosmic Masers: from $\mathrm{OH}$ to $\mathrm{H}_{0}$, ed. R. S. Booth, E. M. L. Humphreys, \& W. H. T. Vlemmings (Cambridge: Cambridge Univ. Press), 54

Vlemmings, W. H. T., Torres, R. M., \& Dodson, R. 2011, A\&A, 529, 95

Watson, W. D. 2009, RMxAC, 36, 113

Wiebe, D. S., \& Watson, W. D. 1998, ApJL, 503, L71 